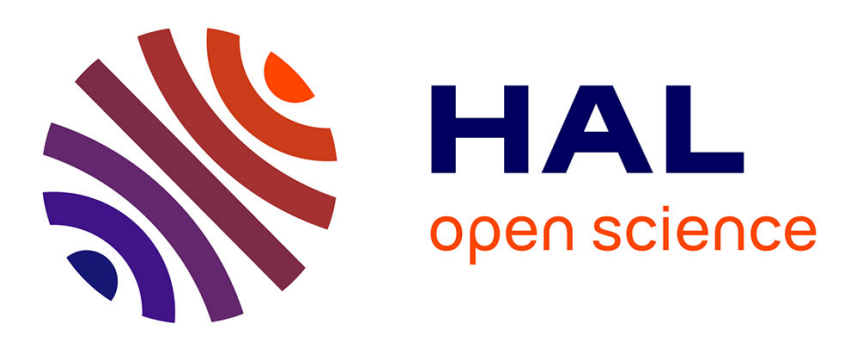

\title{
On the performance of non-conforming finite elements for the upper bound limit analysis of plates
}

Jérémy Bleyer, Patrick de Buhan

\section{To cite this version:}

Jérémy Bleyer, Patrick de Buhan. On the performance of non-conforming finite elements for the upper bound limit analysis of plates. International Journal for Numerical Methods in Engineering, 2013, 94 (3), pp.308-330. 10.1002/nme.4460 . hal-00776908

\section{HAL Id: hal-00776908 \\ https: / hal-enpc.archives-ouvertes.fr/hal-00776908}

Submitted on 18 Jan 2013

HAL is a multi-disciplinary open access archive for the deposit and dissemination of scientific research documents, whether they are published or not. The documents may come from teaching and research institutions in France or abroad, or from public or private research centers.
L'archive ouverte pluridisciplinaire HAL, est destinée au dépôt et à la diffusion de documents scientifiques de niveau recherche, publiés ou non, émanant des établissements d'enseignement et de recherche français ou étrangers, des laboratoires publics ou privés. 


\title{
On the performance of non-conforming finite elements for the upper bound limit analysis of plates
}

\author{
J. Bleyer, P. de Buhan \\ Université Paris-Est, Laboratoire Navier, \\ Ecole des Ponts ParisTech-IFSTTAR-CNRS (UMR 8205) \\ 6-8 av Blaise Pascal, Cité Descartes, \\ 77455 Champs-sur-Marne, FRANCE \\ E-mail: jeremy.bleyer@enpc.fr
}

January 18, 2013

\begin{abstract}
In this paper, the upper bound limit analysis of thin plates in bending is addressed using various types of triangular finite elements for the generation of velocity fields and second order cone programming (SOCP) for the minimization problem. Three different $C^{1}$-discontinuous finite elements are considered : the quadratic 6 node Lagrange triangle (T6), an enhanced T6 element with a cubic bubble function at centroid (T6b) and the cubic Hermite triangle (H3). Through numerical examples involving Johansen and von Mises yield criteria, it is shown that cubic elements (H3) give far better results in terms of convergence rate and precision than fully conforming elements found in the literature, especially for problems involving clamped boundaries.
\end{abstract}

Keywords : limit analysis ; yield design ; upper bound ; thin plates ; displacement model ; finite element method ; second order cone programming

\section{Introduction}

Direct methods to evaluate the limit load of structures without performing a cumbersome incremental elastoplastic analysis become increasingly interesting in most practical engineering problems. Limit analysis, or, in a more general manner, yield design, is one of this powerful tool which exploits two bounding theorems (resp. static and kinematic approach) to bracket the limit load of a structure by respectively a lower and an upper bound. One appealing feature of the yield design approach, is that it only requires the knowledge of a local strength criterion, with no reference to the other aspects of the mechanical behaviour (deformability properties, plastic flow rule, etc...). Its applicability is simply subject to the condition that the geometry changes can be neglected in the analysis. For a complete and general presentation of the yield design theory, the interested reader can refer to [1, 2] and [3] for a specific application to plates and slabs.

The formulation of this method can be applied to all kind of structures (frames, beams, arches, plates, shells,...) and always leads to the resolution of an optimization problem. The static approach leads to maximizing the loading factor over statically admissible stress fields i.e. subjected to equilibrium constraints and fulfillment of the yield criterion at each point of the structure. Various methods have been proposed to solve this problem 4, 5, 6.

The present paper is interested in the dual problem (kinematic approach) which leads to the minimization of a convex non-smooth functional over a set of kinematically admissible virtual velocity fields under a normalization constraint on the power of external work. The finite element method is 
naturally appropriate to solve this kind of problem and an important amount of work can be found in the case of plane strain problems $[6,7,8,9,9$. It should also be mentioned that meshless procedures have recently been applied to plate limit analysis problems with promising results [10, 11, 12.

In addition to a specific discretization procedure, mathematical programming is the other key aspect in numerical limit analysis. Discrete upper bound limit analysis yields a minimization problem involving linear or non-linear programming. Piecewise linearization of yield criteria leads to LP problems but often requires an important number of additional variables. However, most of yield criteria for plates can be formulated as an intersection of cones for which the upper bound problem can be solved as second order cone programming via a primal-dual interior point method implemented in commercial codes such as the Mosek software package 13. This algorithm, proved to be very efficient for limit analysis problems 9 , 10, 11, 14, 15, will be used in our work.

In the case of plate structures, yield line theory has been so far the most commonly used method to obtain analytical or numerical upper bounds by assuming rigid mechanisms separated by hinge or yield lines (usually located on element edges in the case of a finite element implementation). However, this method is highly sensitive to the mesh layout (see [16, 17, 18 and references cited therein). Moreover, as it was pointed out by Braestrup [19, even if exact solutions can be obtained for some problems, yield line theory fails, in general, to predict the exact upper bound even when assuming a very complex mechanism or with an infinitely refined mesh.

In order to obtain good results in the most general case, plastic dissipation through curvature also has to be taken into account, which requires a quadratic interpolation at least of the velocity field. Hodge and Belytschko 20 were the first to obtain interesting lower and upper bounds using finite element representation of velocity and bending moment fields. A discontinuous quadratic displacement field was used in their upper bound formulation and a simplex method was used for the minimization process. Capsoni and Corradi [21] used a $C^{1}$-continuous quadrilateral element (BFS) with 16 degrees of freedom (dof) in conjunction with an iterative algorithm separating rigid zones from the rest of the structure in the minimization procedure. Le [15] adopted a triangular HCT element which is $C^{1}$-continuous (12 dof) with SOCP programming. Both elements yield good results in general but are able to reproduce yield line mechanisms only through localized zones of high curvature.

The main novelty of this paper is to propose the use of efficient $C^{1}$-discontinuous finite elements to perform an upper bound limit analysis. Contrary to elastic computations, the $C^{1}$ continuity is not required in the case of limit analysis, avoiding the highly debated difficulties to construct fully conforming plate elements [22, 23]. Thus, we propose three different finite elements (one with a quadratic and two with a cubic interpolation) to model plastic dissipation through curvature as well as rotation discontinuities.

In section 2 , the general formulation of yield design theory in the case of plates is recalled. The upper bound kinematic approach for plates in bending is more specifically presented. In section 3, the discrete minimization problem obtained after a finite element discretization is detailed and the three finite elements proposed in this paper are presented. Section 4 is devoted to the formulation of the discrete minimization problem in terms of standard second-order cone programming. Finally, various numerical examples will be treated in section 5 to assess the performances of the presented finite elements.

\section{$2 \quad$ Yield design of bending plates : a general background}

The purpose of the present section is to formulate the problem of yield design of plates, first in the most general context, then in the specific case of bending plates, with a special emphasis on the upper bound kinematic approach, based on the virtual work principle. 


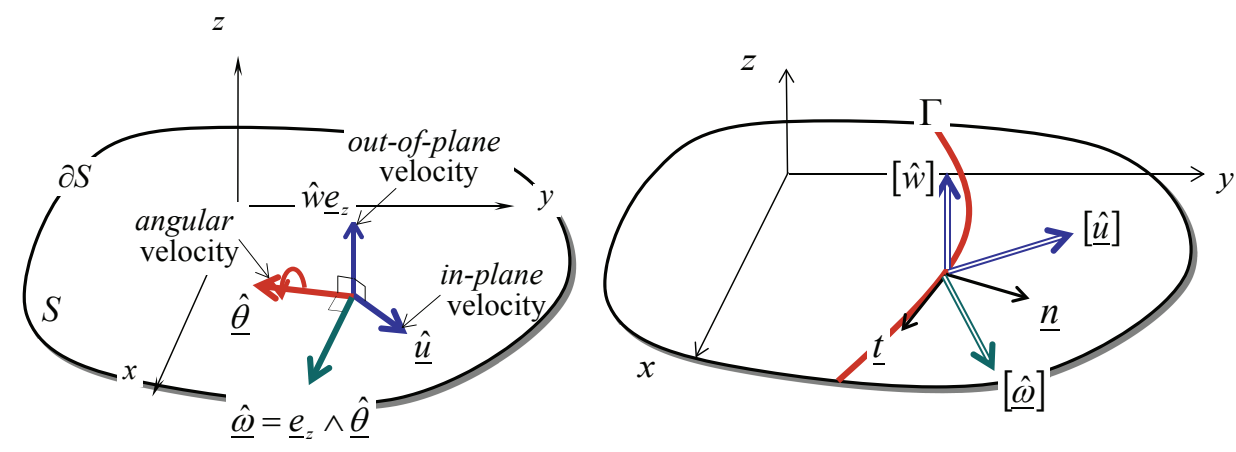

Figure 1: Virtual kinematics of a plate (left) and velocity jumps across a line (right)

\subsection{Virtual motions and virtual work of internal forces for a plate}

Referring to a Cartesian orthonormal frame Oxyz, the plate occupies a two-dimensional geometrical domain $S$ in the $O x y$-plane as shown in Figure 1. The virtual motions of such a plate are defined at any point $(x, y)$ of $S$ by:

- an in-plane velocity $\underline{\widehat{u}}$ along with an out-of-plane (transversal) velocity $\widehat{w} \underline{e}_{z}$ of the particle attached to the point;

- an angular velocity $\underline{\hat{\theta}}$ characterizing the rotation of the microstructure attached to the same point, with the associated vector $\underline{\widehat{\omega}}=\underline{e}_{z} \wedge \underline{\hat{\theta}}$, both vectors being parallel to $S$ (left-hand side of Figure 1).

Following the same line of reasoning as that employed for instance by Salençon [2] for onedimensional beams, making use of the virtual work method and related principles, the virtual work of internal forces may be written in the form :

$$
P_{(i)}(\underline{\widehat{u}}, \widehat{\widehat{w}}, \underline{\widehat{\omega}})=-\int_{S}\left(\underline{\underline{N}}:\left({ }^{s} \underline{\underline{\nabla}}\right)+\underline{V} \cdot(\underline{\nabla \widehat{w}}-\underline{\widehat{\omega}})+\underline{\underline{M}}:\left({ }^{s} \underline{\underline{\nabla}}\right)\right) d S
$$

where ${ }^{s} \underline{\nabla}(\cdot)$ denotes the symmetric part of the gradient operator with respect to the $(x, y)$ coordinates, while $\underline{\underline{\underline{N}}}$ is the tensor of membrane forces, $\underline{V}$ the vector of shear forces and $\underline{\underline{M}}$ the tensor of bending moments. In the case when the virtual velocity fields are discontinuous across a line $\Gamma$ (right-hand side of Figure 1), the above expression (1) should be completed by the following additional line integral:

$$
P_{(i)}^{[]}([\underline{\widehat{u}}],[\widehat{w}],[\underline{\widehat{\omega}}])=-\int_{\Gamma}((\underline{\underline{N}} \cdot \underline{n}) \cdot[\underline{\widehat{u}}]+(\underline{V} \cdot \underline{n})[\widehat{w}]+(\underline{\underline{M}} \cdot \underline{n}) \cdot[\underline{\widehat{\widehat{w}}}]) d \Gamma
$$

where $[*]$ represents the jump of a variable $*$ when crossing $\Gamma$ along its unit normal $\underline{n}$ (Figure 1 ).

\subsection{Statement of the yield design problem for bending plates}

Generally speaking, the strength criterion of a plate can be formulated as a condition involving all internal forces at any point of the plate, namely :

$$
f(\underline{\underline{N}}, \underline{\underline{V}}, \underline{\underline{M}}) \leq 0
$$

The case of the so-called bending plates, under concern in the present paper, corresponds to the situation when the plate is assumed to be infinitely resistant to both shear and membrane forces (and not that such forces can be neglected), so that the above criterion simply reduces to a condition on the sole bending moments :

$$
f(\underline{\underline{M}}) \leq 0
$$


It is assumed from now on, that the plate loading depends upon several parameters $\underline{Q}$, which means that the virtual work of external forces in any kinematically admissible (K.A.) virtual velocity field, may be expressed as:

$$
\forall(\underline{\widehat{u}}, \widehat{\widehat{w}}, \underline{\widehat{\widehat{\omega}}}) \text { K.A. with } \underline{\widehat{\widehat{q}}}, \quad P_{(e)}(\underline{\widehat{\widehat{u}}}, \widehat{\widehat{w}}, \underline{\widehat{\widehat{\omega}}})=\underline{Q} \cdot \underline{\widehat{q}}
$$

where $\underline{\underline{q}}$ represents the generalized kinematic parameters defined by duality in the expression of the work of external forces. In this context, the domain $K$ of potentially safe loads $\underline{Q}$ as introduced in the limit analysis or yield design theory, is defined as follows:

$$
K=\{\underline{Q} ; \exists \underline{\underline{M}} \text { Statically Admissible (S.A.) with } \underline{Q}, \quad \forall(x, y) \in S \quad f(\underline{\underline{M}}(x, y)) \leq 0\}
$$

\subsection{Upper bound kinematic approach}

The upper bound kinematic approach to $K$ is then classically obtained from the virtual work principle

$$
\begin{aligned}
& \forall(\underline{\underline{u}}, \widehat{w}, \underline{\widehat{\omega}}) \text { K.A. with } \underline{\widehat{q}}, \forall(\underline{\underline{N}}, \underline{V}, \underline{\underline{M}}) \text { S.A. with } \underline{Q} \\
& P_{(e)}(\underline{\widehat{u}}, \widehat{w}, \underline{\widehat{\omega}})=\underline{Q} \cdot \underline{\widehat{q}}=-P_{(i)}(\underline{\widehat{\widehat{u}}}, \widehat{\widehat{w}}, \underline{\widehat{\widehat{\omega}}})
\end{aligned}
$$

introducing the so called maximum resisting work (also called maximum plastic dissipation in the context of limit analysis), defined as :

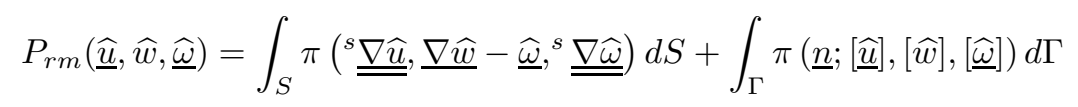

where the support functions are :

$$
\begin{aligned}
& \pi\left({ }^{s} \underline{\underline{\nabla \widehat{u}}}, \underline{\nabla \widehat{w}}-\underline{\widehat{\widehat{\omega}}},{ }^{s} \underline{\underline{\nabla \widehat{\omega}}}\right)=\sup _{(\underline{\underline{N}}, \underline{\underline{\underline{M}}}, \underline{\underline{\underline{M}}})}\left\{\underline{\underline{\underline{N}}}:{ }^{s} \underline{\underline{\nabla \widehat{u}}}+\underline{V} \cdot(\underline{\nabla \widehat{w}}-\underline{\widehat{\widehat{\omega}}})+\underline{\underline{M}}::^{s} \underline{\underline{\nabla \widehat{\omega}}} ; f(\underline{\underline{M}}) \leq 0\right\} \\
& \pi(\underline{\underline{n}} ;[\underline{\widehat{u}}],[\widehat{w}],[\underline{\widehat{\omega}}])=\sup _{(\underline{\underline{N}}, \underline{V}, \underline{\underline{M}})}\{(\underline{\underline{N}} \cdot \underline{n}) \cdot[\underline{\widehat{\widehat{u}}}]+(\underline{V} \cdot \underline{\underline{n}})[\widehat{w}]+(\underline{\underline{M}} \cdot \underline{n}) \cdot[\underline{\widehat{\widehat{\omega}}}] ; f(\underline{\underline{M}}) \leq 0\}
\end{aligned}
$$

It clearly appears from (6) and (17) that, since in the case of bending plates there is no limitation on $\underline{\underline{N}}$ and $\underline{V}$, the support functions and thus the maximum resisting work take finite values if and only $\overline{\overline{\text { if }}}$ the associated strain variables are equal to zero, which implies the following kinematic constraints on the virtual motions:

$$
{ }^{s} \underline{\underline{\nabla \widehat{u}}}=\underline{\underline{0}}, \underline{\nabla \widehat{w}}-\underline{\widehat{\omega}}=\underline{0}
$$

as well as :

$$
[\underline{\widehat{u}}]=\underline{0},[\widehat{w}]=0
$$

It should be observed that the second condition of equation (8) is none other than the well-known Love-Kirchhoff condition leading to the following expression of the strain variable associated to the bending moments:

$$
\stackrel{\underline{\nabla} \underline{\underline{\omega}}}{=} \underline{\underline{\nabla}}(\underline{\nabla \widehat{w}})=\left[\begin{array}{cc}
\frac{\partial^{2} \widehat{w}}{\partial x^{2}} & \frac{\partial^{2} \widehat{w}}{\partial x \partial y} \\
\frac{\partial^{2} \widehat{w}}{\partial x \partial y} & \frac{\partial^{2} \widehat{w}}{\partial y^{2}}
\end{array}\right]=\underline{\underline{\hat{\chi}}}
$$

called the virtual rate of curvature. It follows that (6) simplifies into:

$$
\pi(\underline{\underline{\widehat{\chi}}})=\sup \{\underline{\underline{M}}: \underline{\underline{\hat{\chi}}} ; f(\underline{\underline{M}}) \leq 0\}
$$




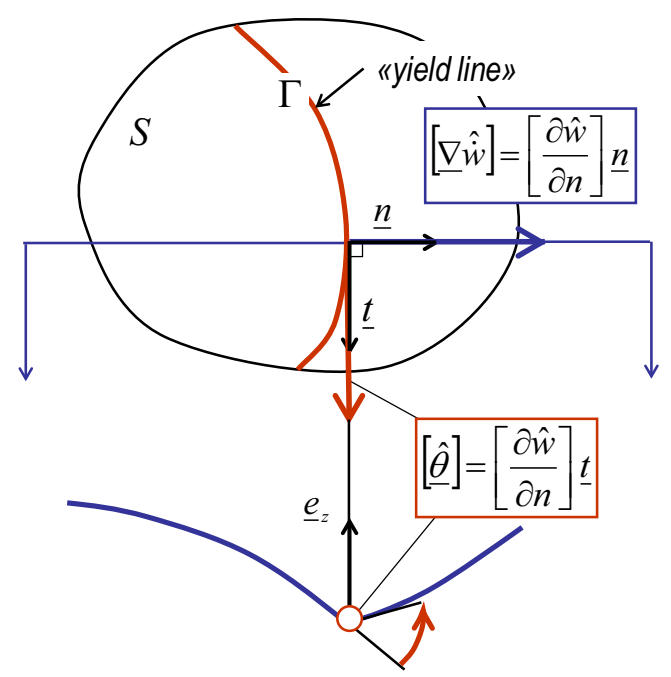

Figure 2: Jump of angular velocity across a yield line

On the other hand, since the virtual transverse velocity must remain continuous (second condition of (9) ), the jump of its gradient across a discontinuity line $\Gamma$ is necessarily normal to this line :

$$
[\underline{\nabla \widehat{w}}]=\left[\frac{\partial \widehat{w}}{\partial n}\right] \underline{n}
$$

while the associated jump of angular velocity is tangent to $\Gamma$ (see Figure 2) :

$$
[\underline{\widehat{\theta}}]=\left[\frac{\partial \widehat{w}}{\partial n}\right] \underline{t} \quad \text { with } \underline{t}=\underline{n} \wedge \underline{e}_{z}
$$

The corresponding support function (7) is therefore reduced to :

$$
\pi(\underline{n} ; \underline{\hat{\theta}}])=\sup \left\{M_{n n}[\underline{\widehat{\theta}}] ; f(\underline{\underline{M}}) \leq 0\right\}
$$

As a consequence, the implementation of the yield design upper bound kinematic approach for bending plates is based upon considering distributions of K.A. transverse velocity fields $\widehat{w}$ subject to the following properties:

- $\widehat{w}$ is everywhere continuous;

- its second gradient (rate of curvature $\underline{\hat{\chi}}$ ) can be calculated everywhere, except on a number of discontinuity lines (yield lines) where jümps of angular velocities have to be taken into account.

For the sake of simplicity, the notation ${ }^{-}$recalling the virtual character of the kinematic fields involved in the analysis will from now on be omitted. Transverse virtual velocity fields $\widehat{w}$ will also be noted $u$ (and called indifferently velocity or displacement fields) in order to have notations consistent with usual finite elements conventions.

\subsection{Support functions for usual strength criteria}

Given a bending moment tensor field $\underline{\underline{M}}$ with principal values $M_{1}, M_{2}$, we consider the following commonly used criteria for thin plate in bending : 
- Johansen : $-m_{p}^{\prime} \leq M_{1}, M_{2} \leq m_{p}$

- Tresca : $\max \left(\left|M_{1}\right|,\left|M_{2}\right|,\left|M_{1}-M_{2}\right|\right) \leq m_{p}$

- von Mises : $M_{1}^{2}+M_{2}^{2}-M_{1} M_{2}-m_{p}^{2} \leq 0$

where $m_{p}$ and $m_{p}^{\prime}$ are positive and negative yield moments. For sake of simplicity, we will work here in the particular case $m_{p}=m_{p}^{\prime}$

For the previously mentioned criteria, expressions of (10) are given by :

- Johansen : $\pi(\underline{\underline{\chi}}(x, y))=m_{p}\left|\chi_{1}\right|+m_{p}\left|\chi_{2}\right|$

- Tresca : $\pi(\underline{\underline{\chi}}(x, y))=m_{p} \cdot \max \left\{\left|\chi_{1}\right|,\left|\chi_{2}\right|,\left|\chi_{1}+\chi_{2}\right|\right\}$

- von Mises : $\pi(\underline{\underline{\chi}}(x, y))=\frac{2 m_{p}}{\sqrt{3}} \sqrt{\chi_{1}^{2}+\chi_{2}^{2}+\chi_{1} \chi_{2}}=\frac{2 m_{p}}{\sqrt{3}} \sqrt{\chi_{x x}^{2}+\chi_{y y}^{2}+\chi_{x x} \chi_{y y}+\chi_{x y}^{2}}$

where $\chi_{1}, \chi_{2}$ are the principal values of the curvature tensor.

Denoting by $\delta \theta_{n}=\left[\frac{\partial u}{\partial n}\right]$ the normal rotation discontinuity, expressions of (11) are given by:

- Johansen : $\pi\left(\delta \theta_{n}, \underline{n}\right)=m_{p}\left|\delta \theta_{n}\right|$

- Tresca : $\pi\left(\delta \theta_{n}, \underline{n}\right)=m_{p}\left|\delta \theta_{n}\right|$

- Von Mises : $\pi\left(\delta \theta_{n}, \underline{n}\right)=\frac{2 m_{p}}{\sqrt{3}}\left|\delta \theta_{n}\right|$

\subsection{Upper bound kinematic formulation}

The plate is assumed to be loaded by a transverse loading distribution $q(x, y) \underline{e}_{z}$ and that its boundary $\partial S$ can be decomposed into four parts $\partial S_{i}$ such that $\partial S=\bigcup \partial S_{i} \forall i=0, \ldots, 3$ with $\partial S_{i} \cap \partial S_{j}=\emptyset$ $\forall i \neq j$ and :

- on $\partial S_{1}: u=0$ (simply supported)

- on $\partial S_{2}: \frac{\partial u}{\partial n}=0$ (fixed rotation, free displacement)

- on $\partial S_{3}: u=0$ and $\frac{\partial u}{\partial n}=0$ (clamped boundary)

- on $\partial S_{0}$ : free boundary

The set of boundary conditions is denoted by $\mathcal{B}$.

The set of kinematically admissible velocity fields is $K A=\{u(x, y) \in V \cap \mathcal{B}\}$ where $V$ is the functional space consistent with the limit analysis of thin plates. Hence, $V$ will be the set of transverse velocity fields which are $C^{0}$-continuous on $S$, piecewise $C^{1}$ and $C^{2}$-continuous on $S \backslash \Gamma$ where $\Gamma$ is a set of yield lines in $S$.

In this case, the work of external forces writes:

$$
P_{(e)}(u)=\int_{S} q(x, y) u(x, y) d S
$$

While the maximum resisting work is $\forall u \in K A$ :

$$
P_{r m}(u)=P_{r m}^{\text {curv }}(u)+P_{r m}^{\text {disc }}(u)=\int_{S} \pi(\underline{\underline{\chi}}(x, y)) d S+\int_{\Gamma} \pi\left(\delta \theta_{n}, \underline{n}\right) d l
$$




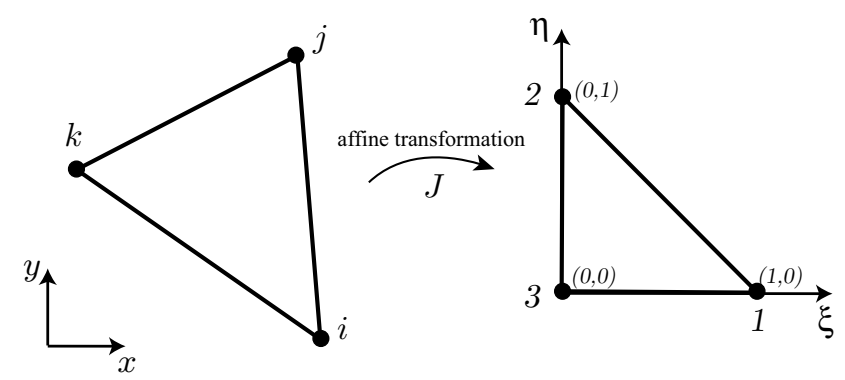

Figure 3: Geometric transformation to reference plane

The kinematic formulation for the ultimate load factor $\lambda$ is then given by the following optimization problem :

$$
\lambda=\min _{\substack{\forall u \in K A \\ P_{(e)}(u)=1}} P_{r m}(u)
$$

\section{Finite element discretization}

Finite element discretization is presented in this section in the case of a von Mises criterion. Extensions to other criteria are discussed in section 4 .

\subsection{Formulation of the discrete problem}

As regards the upper bound limit analysis, finite elements are widely used to discretize the minimization problem (14) by interpolating the displacement field $u$ in each element.

We consider the general case of a triangular finite element $e$ with $s$ degrees of freedom $q_{i}^{(e)}$ and given shape functions $N_{i}(\xi, \eta) \forall i=1, \ldots, s$ defined in the $(\xi, \eta)$-plane of a reference element. We will only consider affine geometric transformation from the local to the reference element (see fig. 3). Interpolation of the displacement $u(\zeta)$ at a given point $\zeta=(\xi, \eta)$ in element $e$ is :

$$
u(\zeta)=\sum_{i=1}^{s} N_{i}(\zeta) q_{i}^{(e)}
$$

We will note $\left[D_{1}\right]$ and $\left[D_{2}\right]$ the matrices of first and second derivatives of the shape functions $N_{i}$ in the reference plane :

$$
\left[D_{1}(\xi, \eta)\right]=\left[\begin{array}{c}
N_{i, \xi}(\xi, \eta) \\
N_{i, \eta}(\xi, \eta)
\end{array}\right] \quad\left[D_{2}(\xi, \eta)\right]=\left[\begin{array}{c}
N_{i, \xi \xi}(\xi, \eta) \\
N_{i, \eta \eta}(\xi, \eta) \\
2 N_{i, \xi \eta}(\xi, \eta)
\end{array}\right]
$$

Matrices $\left[J_{1}\right]$ and $\left[J_{2}\right]$ involving geometric transformation terms are used to obtain derivatives along $x$ and $y$. We have therefore :

$$
\left\{\begin{array}{l}
\partial_{x} u(\zeta) \\
\partial_{y} u(\zeta)
\end{array}\right\}=\left[J_{1}\right]\left[D_{1}(\zeta)\right]\left\{q_{e}\right\} \quad\{\kappa(\zeta)\}=\left\{\begin{array}{c}
\partial_{x x} u(\zeta) \\
\partial_{y y} u(\zeta) \\
2 \partial_{x y} u(\zeta)
\end{array}\right\}=\left[J_{2}\right]\left[D_{2}(\zeta)\right]\left\{q_{e}\right\}
$$

where $\left\{q_{e}\right\}$ collects the $q_{i}^{(e)}$ dof. 


\subsubsection{Contribution of the curvature to the dissipation}

The contribution of element $e$ to the dissipation is for the von Mises criterion :

$$
p_{r m, e}=m_{p} \int_{S_{e}} \sqrt{\{\kappa(\zeta)\}^{T}[Q]\{\kappa(\zeta)\}} d S \quad \text { with } \quad[Q]=\frac{1}{3}\left[\begin{array}{ccc}
4 & 2 & 0 \\
2 & 4 & 0 \\
0 & 0 & 1
\end{array}\right]
$$

These integrals are evaluated by a 2D-Gauss-Legendre quadrature on triangles, involving $n$ Gauss points $\zeta_{g}$ and Gauss weights $\omega_{g} \forall g=1, \ldots, n$. These contributions are summed over all elements to obtain the contribution of the curvature term $P_{r m}^{c u r v}$ to the total dissipation. Letting $\left[B_{e}(\zeta)\right]=$ $\left[J_{2}\right]\left[D_{2}(\zeta)\right]$, we obtain :

$$
P_{r m}^{c u r v}=m_{p} \sum_{e=1}^{N_{e}} \sum_{g=1}^{n} \omega_{g} \operatorname{det}\left[J_{e}\right] \sqrt{\left\langle q_{e}\right\rangle\left[B_{e}\left(\zeta_{g}\right)\right]^{T}[Q]\left[B_{e}\left(\zeta_{g}\right)\right]\left\{q_{e}\right\}}
$$

where $N_{e}$ is the total number of elements and $\operatorname{det}\left[J_{e}\right]$ the jacobian of the geometric transformation for $e$.

\subsubsection{Contribution of rotation discontinuities to the dissipation}

We consider two adjacent elements $e$ and $e^{\prime}$ along edge $j$. Letting $\left\langle n_{j}\right\rangle=\left[\begin{array}{ll}n_{j x} & n_{j y}\end{array}\right]$ be the coordinates of a unit normal vector of edge $j$, absolute value of the normal rotation discontinuity at a point $\tau$ on $j$ will be :

$$
\left|\delta \theta_{n}(\tau)\right|=\left|\left\langle n_{j}\right\rangle\left[J_{1}^{(e)}\right]\left[D_{1}^{(e)}(\tau)\right]\left\{q_{e}\right\}-\left\langle n_{j}\right\rangle\left[J_{1}^{\left(e^{\prime}\right)}\right]\left[D_{1}^{\left(e^{\prime}\right)}(\tau)\right]\left\{q_{e^{\prime}}\right\}\right|=\left|\left\langle\delta \Theta_{j}(\tau)\right\rangle\{U\}\right|
$$

where this expression is collected into a global line vector $\left\langle\delta \Theta_{j}\right\rangle$ and where $\{U\}$ is the global vector of degrees of freedom.

The contribution of edge $j$ to the dissipation is then :

$$
p_{r m, j}=\int_{\Gamma_{j}} \frac{2 m_{p}}{\sqrt{3}}\left|\left\langle\delta \Theta_{j}(\tau)\right\rangle\{U\}\right| d \tau
$$

These integrals are also evaluated via a 1D-Gauss-Legendre quadrature along line $\Gamma_{j}$ with $m$ Gauss points $\tau_{g^{\prime}}$ and weights $w_{g^{\prime}} \forall g^{\prime}=1, \ldots, m$. These contributions are summed over all $N_{d}$ active edges to obtain the total contribution $P_{r m}^{\text {disc }}$ of the rotation discontinuities to the dissipation :

$$
P_{r m}^{d i s c}=\frac{2 m_{p}}{\sqrt{3}} \sum_{j=1}^{N_{d}} \sum_{g^{\prime}=1}^{m} w_{g^{\prime}} \operatorname{det}\left[J_{j}\right]\left|\left\langle\delta \Theta_{j}\left(\tau_{g^{\prime}}\right)\right\rangle\{U\}\right|
$$

where $\operatorname{det}\left[J_{j}\right]$ is the jacobian of the geometric transformation for $j$.

Remark : Active edges represent edges which can potentially contribute to $P_{r m}$ via a rotation discontinuity. This involves internal edges of the mesh and also edges belonging to the $\partial S_{2} \cup \partial S_{3}$ part of the boundary where the normal rotation is set equal to zero.

\subsubsection{Power of external loads}

The power of external loads can be computed analytically or numerically by evaluating $\forall e=1, \ldots, N_{e}$, $\forall i=1, \ldots, s$ :

$$
f_{e, i}=\int_{S_{e}} N_{i}(\zeta) q(\zeta) d S
$$

which will be collected into a global vector $\{F\}=\left\{\left(f_{e, i}\right)\right\}$ such that $P_{\text {ext }}=\{F\}^{T} \cdot\{U\}$. 


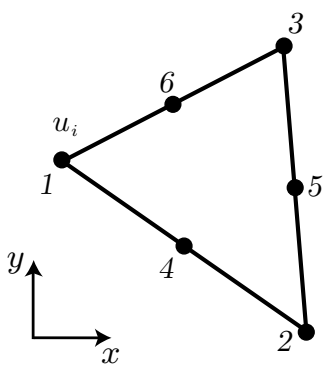

Figure 4: T6 finite element

\subsubsection{Discrete minimization problem}

Finally, the discrete minimization problem can be expressed as :

$$
\begin{gathered}
\lambda=\min _{\{U\}} m_{p} \sum_{e=1}^{N_{e}} \sum_{g=1}^{n} \omega_{g} \operatorname{det}\left[J_{e}\right] \sqrt{\left\langle q_{e}\right\rangle\left[B_{e}\right]^{T}[Q]\left[B_{e}\right]\left\{q_{e}\right\}}+\frac{2 m_{p}}{\sqrt{3}} \sum_{j=1}^{N_{d}} \sum_{g^{\prime}=1}^{m} w_{g^{\prime}} \operatorname{det}\left[J_{j}\right]\left|\left\langle\delta \Theta_{j}\right\rangle\{U\}\right| \\
\text { subject to }\{F\}^{T} \cdot\{U\}=1
\end{gathered}
$$

with appropriate boundary conditions which can be taken into account during the assembling process of the global vectors.

\section{$3.2 \quad$ T6 element}

Pure yield line elements were frequently proposed in the literature [24, 25. These elements are actually Lagrange triangles with 3 nodes (T3). Since linear interpolation of the displacement is assumed, no curvature is developed in this element and dissipation can only appear through normal rotation discontinuity along edges. It should be stressed that, contrary to elastic finite element computations, rotation discontinuities are perfectly admissible for plate limit analysis problems, since such displacement fields are kinematically admissible in the sense of subsection 2.3. Therefore, non-conformity is only a technical term employed to designate finite elements with discontinuous normal derivatives. In order to obtain better performances, we first propose to compute the previous quantities by considering the quadratic Lagrange triangle with 6 nodes (figure (4). This nonconforming element gives linear varying rotations along edges and a constant curvature field throughout the element.

Since the curvature field is constant, we can choose only $n=1$ Gauss point to evaluate $P_{r m}^{\text {curv }}$. We will also choose, in the following, $m=3$ Gauss points over each edge to approximate $P_{r m}^{\text {disc }}$. Expressions of shape functions and derivative matrix are given in Appendix A.

\subsection{T6b element}

The second considered element we is an enhanced version of the preceding T6 element called T6b. A seventh degree of freedom is added by considering the displacement $u_{g}$ at the centroid in addition to the 6 nodal displacements of the T6 element (Fig. 5). The associated shape function

$$
N_{7}(\xi, \eta)=27 \xi \eta(1-\xi-\eta)
$$

is a cubic bubble function such that $N_{7}(\xi, \eta)=0$ on the boundary $\partial S_{e}$ of the element and is equal to 1 at centroid $(\xi=1 / 3, \eta=1 / 3)$.

As a consequence, this element is still non-conforming, the interpolation of the displacement is an 


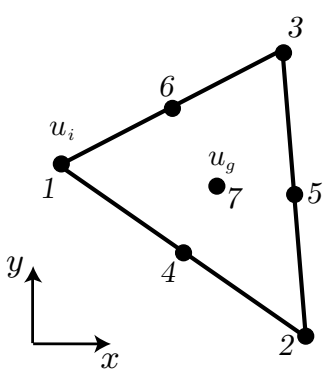

Figure 5: T6b finite element

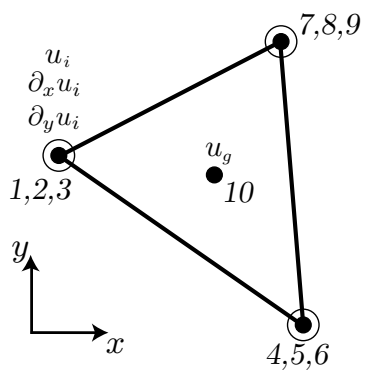

Figure 6: H3 finite element

incomplete cubic expansion, normal rotation is quadratic and the curvature field is linear. We will choose $n=m=3$ for the Gauss rules.

\section{$3.4 \quad$ H3 element}

The third element considered in this paper is the cubic Hermite triangle (H3) represented in Figure 6. Our motivation is to explore the performance of a complete cubic expansion for the transverse displacement contrary to the previous incomplete expansion. The Hermite triangle will be preferred to the cubic Lagrange triangle in order to take advantage of the reduction of the global number of dof when considering dof placed at nodes instead of dof placed on edges.

This nonconforming element has 10 dof : the transverse displacement and the two rotations at corner nodes plus the transverse displacement at centroid. In this case, normal rotations are also quadratic along edges and the curvature field is linear. We will choose $n=m=3$ as well.

Remark : Due to the Hermite nature of this element, special treatment for curved boundaries has to be taken into account. This treatment is discussed in Appendix B.

\section{Mathematical programming}

In this section, the formulation of the problem as second order cone programming problems is considered. 


\subsection{Standard second order cone programming (SOCP)}

The standard form of a second order cone programming problem can be expressed as the following minimization problem of a linear objective function under conic constraints [13] :

$$
\begin{array}{lc}
\min & \{c\} \cdot\{x\} \\
\text { s.t. } & \left\{b_{l}\right\} \leq[A]\{x\} \leq\left\{b_{u}\right\} \\
& \\
& \{x\} \in \mathcal{C}
\end{array}
$$

where $\mathcal{C}$ represents a set of cones $\mathcal{C}_{i}$ on some part $z$ of the components in $\{x\}$. These cones can be :

- the real set : $\mathcal{C}_{i}=\mathcal{C}_{0}=\left\{z \in \mathbb{R}^{k}\right\}$

- the quadratic cone : $\mathcal{C}_{i}=\mathcal{C}_{q}=\left\{z \in \mathbb{R}^{k+1}: z_{1} \geq \sqrt{\sum_{j=2}^{k+1} z_{j}^{2}}\right\}$

- the rotated quadratic cone $: \mathcal{C}_{i}=\mathcal{C}_{r}=\left\{z \in \mathbb{R}^{k+2}: 2 z_{1} z_{2} \geq \sqrt{\sum_{j=3}^{k+2} z_{j}^{2}} \quad z_{1}, z_{2} \geq 0\right\}$

\subsection{Formulation for the Von Mises criterion}

In order to cast problem (17) as a second order cone programming problem, we introduce the Cholseky factor $[C]$ of matrix $[Q]$ such that $[C]^{T}[C]=[Q]$ :

$$
[C]=\frac{1}{\sqrt{3}}\left[\begin{array}{ccc}
2 & 1 & 0 \\
0 & \sqrt{3} & 0 \\
0 & 0 & 1
\end{array}\right]
$$

Letting $\left[R_{e, g}\right]$ such that $\left[R_{e, g}\right]\{U\}=[C]\left[B_{e}\left(\zeta_{g}\right)\right]\left\{q_{e}\right\}$, problem (17) can be rewritten :

$$
\lambda=\min _{\{U\}} m_{p} \sum_{e=1}^{N_{e}} \sum_{g=1}^{n} \omega_{g} \operatorname{det}\left[J_{e}\right]\left\|\left[R_{e, g}\right]\{U\}\right\|+\frac{2 m_{p}}{\sqrt{3}} \sum_{j=1}^{N_{d}} \sum_{g^{\prime}=1}^{m} w_{g^{\prime}} \operatorname{det}\left[J_{j}\right]\left|\left\langle\delta \Theta_{j}\left(\tau_{g^{\prime}}\right)\right\rangle\{U\}\right|
$$

with $\{F\}^{T} \cdot\{U\}=1$ and $\|\cdot\|$ being the standard euclidean norm.

Finally, introducing auxiliary variables $\left\{r_{k}\right\} \in \mathbb{R}^{3}, t_{k} \in \mathbb{R} \forall k=1, \ldots, N_{e} \cdot n$ and $s_{h}, v_{h} \in \mathbb{R} \forall h=$ $1, \ldots, N_{d} \cdot m$, we have :

$$
\begin{array}{lll}
\min _{\{x\}} & m_{p}\left(\sum_{k=1}^{N_{e} \cdot n} c_{k} t_{k}+\sum_{h=1}^{N_{d} \cdot m} c_{h}^{\prime} s_{h}\right) & \\
\text { s.t. } & \{F\}^{T} \cdot\{U\}=1 & \\
& \left\{r_{k}\right\}=\left[R_{e, g}\right]\{U\} & \forall e=1, \ldots, N_{e} \quad \forall g=1, \ldots, n \\
& t_{k} \geq\left\|\left\{r_{k}\right\}\right\| & \forall k=1, \ldots, N_{e} \cdot n \\
& v_{h}=\left\langle\delta \Theta_{j}\left(\tau_{g^{\prime}}\right)\right\rangle\{U\} & \forall j=1, \ldots, N_{d} \quad \forall g^{\prime}=1, \ldots, m \\
& s_{h} \geq\left|v_{h}\right| & \forall h=1, \ldots, N_{d} \cdot m
\end{array}
$$

with $c_{k}=\omega_{g} \operatorname{det}\left[J_{e}\right]$ and $c_{h}^{\prime}=\frac{2}{\sqrt{3}} w_{g^{\prime}} \operatorname{det}\left[J_{j}\right]$. This is a standard second order cone programming form involving equality, inequality and quadratic cone constraints. The total number of variables of optimization collected in vector $\{x\}$ (including $\{U\}$ and auxiliary variables) is $N=N_{q}+4 \cdot n \cdot N_{e}+$ $2 \cdot m \cdot N_{d}$ where $N_{q}$ is the global number of kinematic degrees of freedom. 


\subsection{Formulation for the Johansen criterion}

In the case of a Johansen criterion, the $\pi$ - function can be rewritten as [26] :

$$
\pi(\underline{\underline{\chi}})=m_{p}\left(\chi_{x x}^{+}+\chi_{y y}^{+}\right)+m_{p}^{\prime}\left(\chi_{x x}^{-}+\chi_{y y}^{-}\right)
$$

with

$$
\left\{\begin{array}{c}
\chi_{x x} \\
\chi_{y y} \\
\chi_{x y}
\end{array}\right\}=\left[\begin{array}{cccccc}
1 & 0 & 0 & -1 & 0 & 0 \\
0 & 1 & 0 & 0 & -1 & 0 \\
0 & 0 & \sqrt{2} & 0 & 0 & \sqrt{2}
\end{array}\right]\left\{\begin{array}{c}
\chi_{x x}^{+} \\
\chi_{y y}^{+} \\
\chi_{x y}^{+} \\
\chi_{x x}^{-} \\
\chi_{y y}^{-} \\
\chi_{x y}^{-}
\end{array}\right\} \text {and }\left\{\begin{array}{c}
\chi_{x x}^{+} \\
\chi_{y y}^{+} \\
\chi_{x y}^{+}
\end{array}\right\},\left\{\begin{array}{c}
\chi_{x x}^{-} \\
\chi_{y y}^{-} \\
\chi_{x y}^{y}
\end{array}\right\} \in \mathcal{C}_{r}
$$

where $\mathcal{C}_{r}$ is the rotated quadratic cone. We note $[P]$ the preceding $3 \times 6$ matrix.

Here, with $m_{p}=m_{p}^{\prime}$, we have :

$$
\begin{aligned}
& P_{r m}^{\text {curv }}=m_{p} \sum_{e=1}^{N_{e}} \sum_{g=1}^{n} \omega_{g} \operatorname{det}\left[J_{e}\right]\left\langle\begin{array}{llllll}
1 & 1 & 0 & 1 & 1 & 0\rangle
\end{array}\left\{\begin{array}{l}
\left\{\chi_{e, g}^{+}\right\} \\
\left\{\chi_{e, g}^{-}\right\}
\end{array}\right\}\right. \\
& \text {with } \begin{cases}{\left[B_{e}\left(\zeta_{g}\right)\right]\left\{q_{e}\right\}=[P]\left\{\begin{array}{l}
\left\{\chi_{e, g}^{+}\right\} \\
\left\{\chi_{e, g}^{-}\right\}
\end{array}\right\}} & \forall e=1, \ldots, N_{e} \\
\left\{\chi_{e, g}^{+}\right\},\left\{\chi_{e, g}^{-}\right\} \in \mathcal{C}_{r} & \forall g=1, \ldots, n\end{cases}
\end{aligned}
$$

Since the discontinuity term differs only from a factor $\frac{2}{\sqrt{3}}$, we have :

$$
P_{r m}^{\text {disc }}=m_{p} \sum_{j=1}^{N_{d}} \sum_{g^{\prime}=1}^{m} w_{g^{\prime}} \operatorname{det}\left[J_{j}\right]\left|\left\langle\delta \Theta_{j}\left(\tau_{g^{\prime}}\right)\right\rangle\{U\}\right|
$$

Hence, the minimization problem corresponding to a Johansen criterion can also be written as a standard SOCP problem :

$$
\begin{array}{lll}
\min _{\{x\}} & m_{p}\left(\sum_{k=1}^{N_{e} \cdot n}\left\langle c_{k}\right\rangle\left\{r_{k}\right\}+\sum_{h=1}^{N_{d} \cdot m} c_{h}^{\prime} s_{h}\right) & \\
\text { s.t. } & \{F\}^{T} \cdot\{U\}=1 & \\
& {[P]\left\{r_{k}\right\}=\left[B_{e}\left(\zeta_{g}\right)\right]\left\{q_{e}\right\}} & \forall e=1, \ldots, N_{e} \quad \forall g=1, \ldots, n \\
& \left\{r_{k}\right\}_{1 \rightarrow 3},\left\{r_{k}\right\}_{4 \rightarrow 6} \in \mathcal{C}_{r} & \forall k=1, \ldots, N_{e} \cdot n \\
& v_{h}=\left\langle\delta \Theta_{j}\left(\tau_{g^{\prime}}\right)\right\rangle\{U\} & \forall j=1, \ldots, N_{d} \quad \forall g^{\prime}=1, \ldots, m \\
& s_{h} \geq\left|v_{h}\right| & \forall h=1, \ldots, N_{d} \cdot m
\end{array}
$$

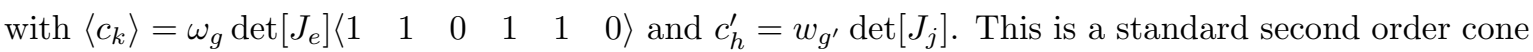
programming form involving equality, inequality and rotated quadratic cone constraints. The total number of variables of optimization is $N=N_{q}+6 \cdot n \cdot N_{e}+2 \cdot m \cdot N_{d}$.

\subsection{Some remarks}

It should be stressed that a SOCP formulation involves auxiliary variables in the optimization process. Performance of the different finite elements will, therefore, be compared by considering the total number of minimization variables $N$ instead of the mesh size.

It is also to be seen that $N$ depends on the choice made in the quadrature rule for $n$ and $m$. Hence, considering higher interpolation order finite elements will not only increase the number of kinematic dofs $N_{q}$ for a fixed mesh but also require more Gauss points to evaluate integrals with sufficient accuracy.

For the same reason, the use of macroelements such as HCT 27, involving three sub-triangles to ensure the $C^{1}$-continuity, will also lead to a higher number of variables $N$ since integration must be performed over each sub-triangle despite omitting the discontinuity terms. 


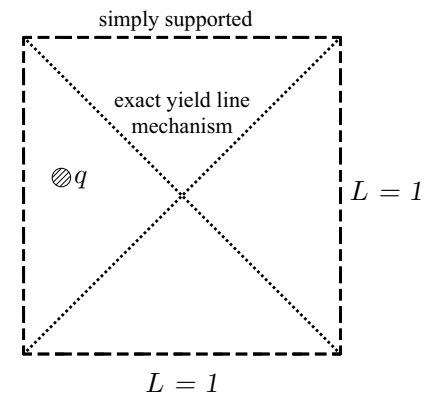

Figure 7: Simply supported square plate with exact collapse mechanism involving diagonal yield lines

\begin{tabular}{clc|c|c|c}
\hline \multicolumn{2}{c|}{ Mesh } & T3 & T6 & T6b & H3 \\
\hline \multirow{3}{*}{ Mesh A } & $M=4$ & $24(0)$ & $24(0)$ & $24(0)$ & $26.63(11)$ \\
& $M=8$ & $24(0)$ & $24(0)$ & $24(0)$ & $24.92(3.9)$ \\
& $M=16$ & $24(0)$ & $24(0)$ & $24(0)$ & $24.43(1.8)$ \\
\hline \multirow{3}{*}{ Mesh B } & $M=4$ & $44.31(84.6)$ & $44.31(84.6)$ & $31.17(29.9)$ & $28.31(18)$ \\
& $M=8$ & $44.31(84.6)$ & $44.3(84.6)$ & $29.77(24)$ & $26.92(12.2)$ \\
& $M=16$ & $44.31(84.6)$ & $44.15(83.9)$ & $27.85(16.1)$ & $25.8(7.5)$ \\
\hline \multirow{3}{*}{ Mesh C } & $M=4$ & $35.56(48.1)$ & $35.56(48.1)$ & $28.84(20.2)$ & $28.03(16.8)$ \\
& $M=8$ & $35.56(48.1)$ & $35.56(48.1)$ & $27.41(14.2)$ & $26.12(8.8)$ \\
& $M=16$ & $35.54(48.1)$ & $34.87(45.3)$ & $26.19(9.1)$ & $25.15(4.8)$ \\
\hline \multirow{3}{*}{ Mesh D } & $M=4$ & $29.24(21.8)$ & $29.24(21.8)$ & $26.64(11)$ & $26.09(8.7)$ \\
& $M=8$ & $28.41(18.4)$ & $28.41(18.4)$ & $25.49(6.2)$ & $25.19(5)$ \\
& $M=16$ & $29.24(21.8)$ & $29.24(21.8)$ & $25.32(5.5)$ & $24.75(3.1)$ \\
\hline
\end{tabular}

\section{$5 \quad$ Numerical examples}

In this section the numerical performance of these three nonconforming finite elements is investigated on uniformly loaded plate problems in bending. The discretization procedure is developed in a Matlab environment (version 7.11) and the SOCP minimization problems are solved using Mosek (version 6.0) on a $1.33 \mathrm{GHz}$ Intel i3 running Windows 7. A pure yield line element (T3) was also developed in the same environment. Limit loads will be given in a dimensionless form i.e. as $\lambda=\frac{q L^{2}}{m_{p}}$ where $q$ is the magnitude of the uniform load and $L$ is a characteristic dimension of the plate.

\subsection{Simply supported square plate with a Johansen criterion}

The first example is a simply supported square plate obeying a Johansen criterion. The exact limit load for this problem is known to be $\lambda=24$ corresponding to a yield line mechanism along the diagonals of the plate (Fig. 7).

As already mentioned, the performance of pure yield line elements highly depends on the mesh layout: structured mesh along the diagonal direction will give exactly the correct bound whereas a structured mesh along the opposite direction will give a very crude estimation of the true bound. We will investigate this effect for our elements by considering three structured meshes (A,B and C) and one unstructured mesh (D) with $M=4,8$ and 16 elements along each side (fig. 8). Results for each finite element type are summarized in table 5.1. Relative errors in percent are indicated in parenthesis.

Results indicate that for mesh A, all finite elements give good results. Layout of mesh A has been clearly chosen to reproduce the exact diagonal mechanism along element edges. Since H3 is not fully 


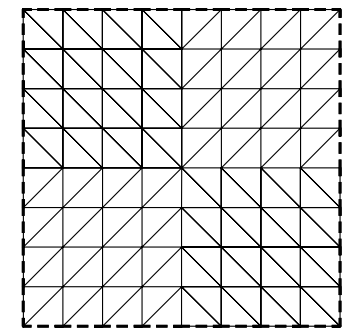

Mesh A

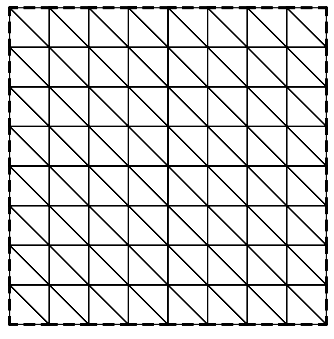

Mesh C

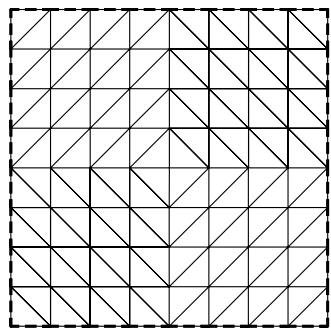

Mesh B

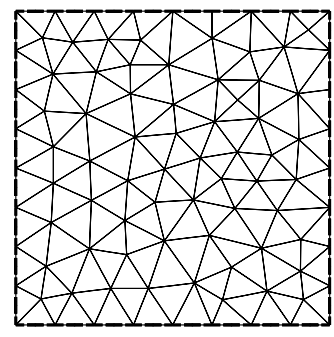

Mesh D

Figure 8: Considered meshes represented for $M=8$

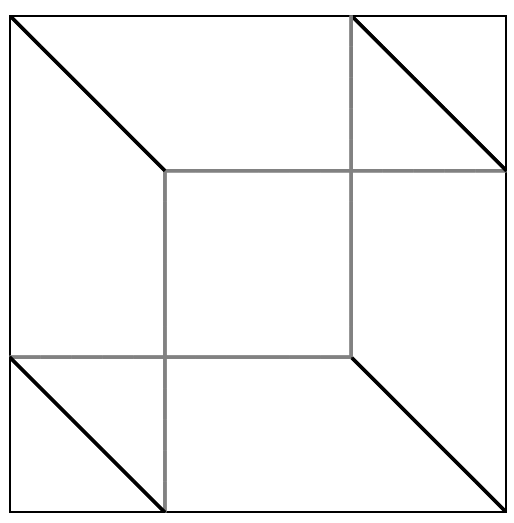

Figure 9: Collapse mechanism obtained with T3 and T6 for mesh C 


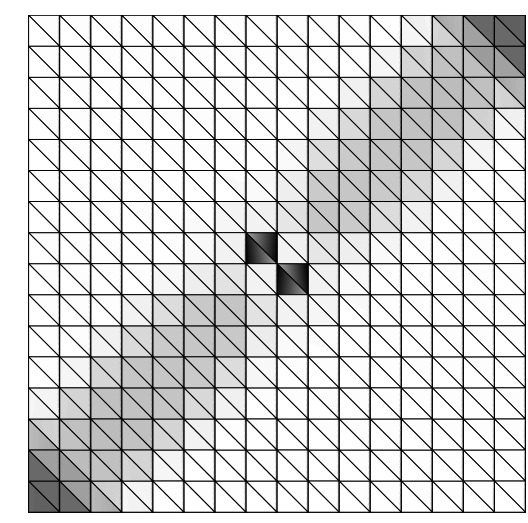

Figure 10: Relative curvature norm distribution obtained with T6b for mesh $\mathrm{C}$

$C^{1}$-discontinuous (derivatives are continuous at nodes only), this element cannot reproduce exactly the failure mechanism as can do T3, T6 and T6b but still gives good results.

Meshes $\mathrm{B}$ and $\mathrm{C}$ have been chosen to avoid reproducing the exact mechanism along element edges. In these cases, T3 and T6 give very poor results which cannot even be improved by mesh refinement. To be more specific, the same mechanism is always obtained for both elements (see Fig. 9 for mesh C). On the contrary, for T6b and H3, even if the obtained bounds are relatively poor for coarse meshes, they are quite rapidly improving with mesh refinement. In Figure 10, we plotted the relative norm of the curvature distribution obtained with T6b for mesh $\mathrm{C}$. We can observe that in the zones where elements are correctly oriented there is no curvature and dissipation is produced only through rotation discontinuities whereas in zones with improper element orientation dissipation is produced through curvature.

The same kind of observation can be made for mesh D for which T3 and T6 are unable to provide a good estimate of the limit load (20\% higher than the exact limit load) whereas T6b and H3 give reasonably good estimates ( $5.5 \%$ and $3.1 \%$ of error for the finest mesh).

\subsection{Clamped square plate with a Johansen criterion}

An exact solution for a uniformly loaded clamped square plate obeying a Johansen criterion has been determined by Fox [28] and involves a quite complex failure mechanism. The corresponding exact limit load is $\lambda^{*}=42.851$.

Unstructured meshes of type D were considered for the different finite elements. In the case of T3 and T6 elements, poor results were obtained (15\% higher than the exact limit load) and it was impossible to improve them by mesh refinement. In the case of T6b elements, a limit load of $\lambda=44.03$ (relative error of $2.7 \%$ ) was obtained with a 444 elements mesh. For the same mesh with H3 elements, we obtained a limit load of $\lambda=43.45$ (relative error of $1.4 \%$ ). Considering the complexity of the exact solution, T6b and H3 elements give very good results for this specific problem.

\subsection{Clamped square plate with a von Mises criterion}

The third example will be a square plate obeying a von Mises criterion with clamped boundaries. There is no known exact solution for this problem, but numerical estimates are summarized in table 1 including upper bounds, lower bounds and approximations from mixed methods.

The best upper bound obtained via a finite element discretization is the one computed by Le [15] with conforming HCT elements : $\lambda^{*}=45.12$. We will compare the results obtained with our elements 
Table 1: Numerical estimates of the limit load of a Von Mises clamped square plate

\begin{tabular}{cccc}
\hline Authors & Type of bound & Type of element & $\lambda$ \\
\hline Hodge and Belytschko [20] & upper & quadratic field (nonconforming) & 49.25 \\
Capsoni and Corradi [21] & upper & BFS (conforming) & 45.29 \\
Le [10] & upper (approximate) & EFG method & 45.07 \\
Le [15] & upper & HCT (conforming) & 45.12 \\
\hline Hodge and Belytschko [20] & lower & quadratic field & 42.86 \\
Le [10] & lower (approximate) & EFG method & 43.85 \\
\hline Andersen et al. [29] & approximation & mixed elements & 44.13
\end{tabular}

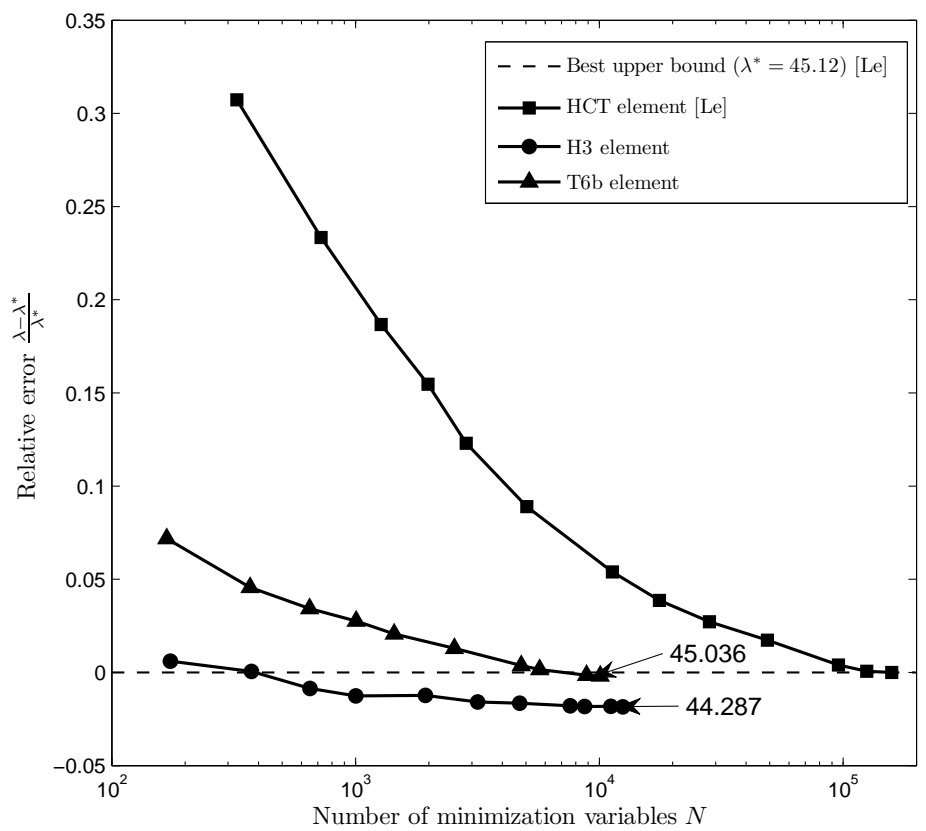

Figure 11: Relative error to the best known upper bound for the clamped square plate problem 

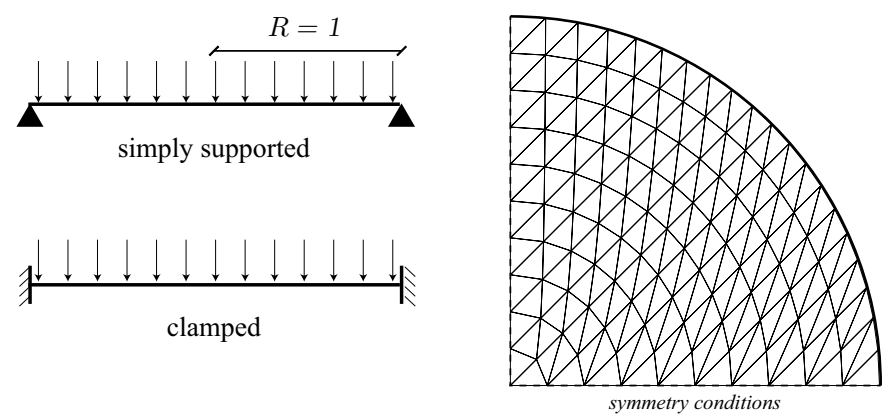

Figure 12: Simply supported and clamped circular plate problem with considered mesh with $M=10$ elements along radii

to this solution for a structured mesh of type A (Fig. 8). Only a quarter of plate was considered with appropriate symmetry conditions.

The relative difference $\left(\lambda-\lambda^{*}\right) / \lambda^{*}$ is plotted against the total number of optimization variables $N$ in Figure 11. In the case of HCT element, we have $N=N_{q}+12 \cdot n \cdot N_{e}$. Since we did not implement the HCT element, the number of mechanical dofs $N_{q}=N_{q, H C T}$ was approximated by taking $N_{q, H C T}=N_{q, H 3}+N_{d}-N_{e}$ since HCT has three dofs per node and one per element edge, whereas H3 has three dofs per node and one per element.

Results of Figure 11 clearly indicate that both elements T6b and H3 improve the best known upper bound for the clamped square plate. We obtain a value of $\lambda=45.036$ for T6 b element (improvement of $0.2 \%$ ) and a value of $\lambda=44.287$ for $\mathrm{H} 3$ element (improvement of $1.85 \%$ ). We can also remark that these values are obtained for a total number of variables $N$ approximately ten times smaller (meshes approximately twice coarser) than the one required to attain $\lambda^{*}$ with HCT elements.

Results for T6 element were not plotted. We observed, indeed, that this element was only able to slightly improve the results given by a yield line element. For instance, for a quarter of plate discretized by 20 elements along each edge, we obtained a limit load of $\lambda=53.23$ with T6 and a limit load of $\lambda=55.43$ with a yield line element.

Recalling the fact that HCT is a quite complex element with cubic interpolation of the displacement over three subtriangles, this example clearly illustrates the benefit of considering nonconforming finite elements for which dissipation can occur through curvature as well as rotation discontinuity. Nevertheless, the poor results given by the T6 element suggest that one has to consider at least a cubic interpolation for the displacement in order to obtain very good results. It is interesting to highlight the important improvement of the results given by the T6b element compared to $\mathrm{T} 6$ by only considering one additional dof associated with one cubic shape function.

\subsection{Circular plate with a von Mises criterion}

Further investigation of the performance of nonconforming finite elements is undertaken by considering the classical problem of a uniformly loaded circular plate with simple and clamped supports (Fig. 12).

Exact solutions for the loading factor are known to be $\lambda^{*}=\frac{q^{*} R^{2}}{m_{p}}=6.516$ for the simply supported case and $\lambda^{*}=12.5$ for the clamped case (where $R$ is the radius of the plate) 30.

Convergence results for the HCT element for the clamped case were also available in the work of Le. Limit loads for the T6b and the H3 element were obtained considering exactly the same structured mesh used in 15. In Figure 13 the relative error to exact load $\lambda^{*}=12.5$ is plotted for the three 


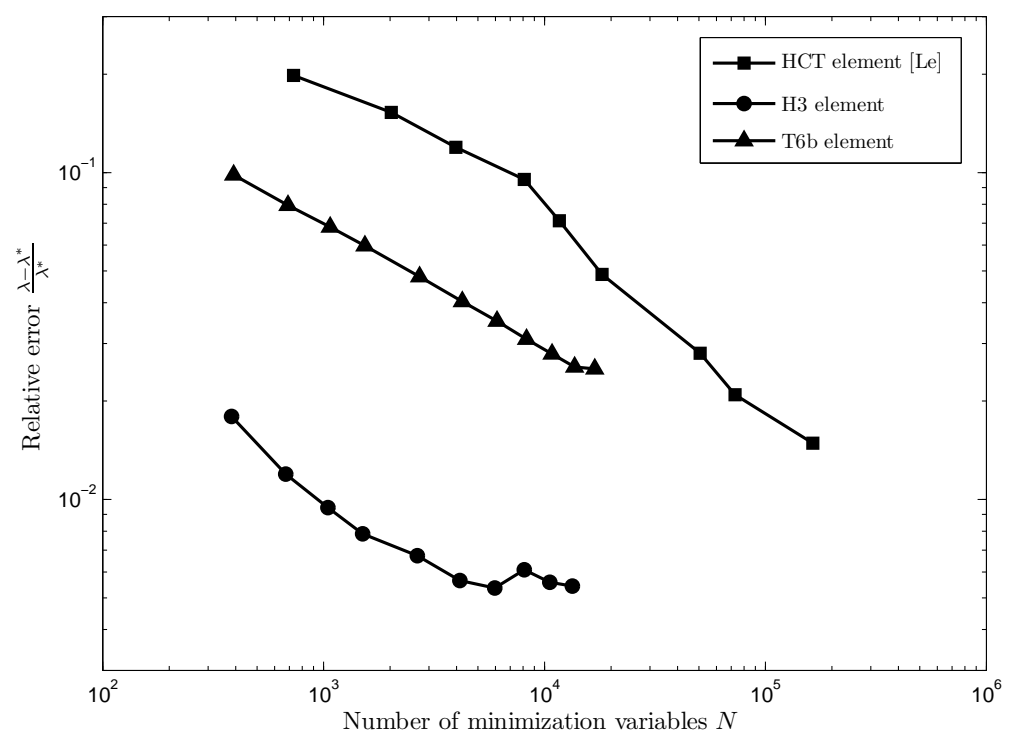

Figure 13: Relative error for the clamped circular plate

elements with a logarithmic scale. Computations for H3 and T6b were performed for meshes up to 800 elements (corresponding to 20 elements along each radius) whereas results for HCT were available for meshes up to 4050 elements (45 elements along each radius).

Results indicate that for the same number of minimization variables H3 is much more accurate than T6b which is itself more accurate than HCT. For the finest meshes in each case, we obtained a limit load of $\lambda=12.68$ for $\mathrm{HCT}$ ( $1.5 \%$ of error), $\lambda=12.81$ for T6b (2.5\% of error) and $\lambda=12.57$ for H3 ( $0.5 \%$ of error). One can also remark that convergence rate seems to be higher in the case of $\mathrm{H} 3$ than T6b or HCT. For the last computed points for H3, difficulties were encountered in the optimization process with Mosek, certainly due to the fact that we were already close to the solution for not very fine meshes.

The case with simply supported supports has also been investigated despite the fact that results with HCT were not available in this case. The errors obtained with T6, T6b and H3 have been compared (Figure 14).

These results clearly underline the difference in the convergence rate for the three different elements. First, it seems, once again, that the T6 element does not converge to the exact limit load but to another higher one $\left(\lambda=7.28\right.$ approximately), hence the convergence rate is very small (in $\left.O\left(N^{0.02}\right)\right)$. In this case, the T6b element converges to the exact solution with a rate in $O\left(N^{0.4}\right)$. The best load factor was found to be $\lambda=6.67$ (2.4\% of error). Finally, the H3 element has the highest convergence rate, being twice the one for T6b (in $\left.O\left(N^{0.8}\right)\right)$. The lowest obtained load factor was $\lambda=6.53(0.2 \%$ of error). We must remark that the number of variables involved for both elements are almost the same for the same mesh size which shows that $\mathrm{H} 3$ element is a very performing element considering accuracy, convergence rate and computational cost.

\subsection{L-shaped plate with a von Mises criterion}

Finally, the case of an L-shaped plate which was also treated in Le [15] is considered. Here again, performances of the T6b, H3 and HCT elements have been compared on the same meshes.

For this problem, no exact solution is known but a very good upper bound is obtained with the yield 


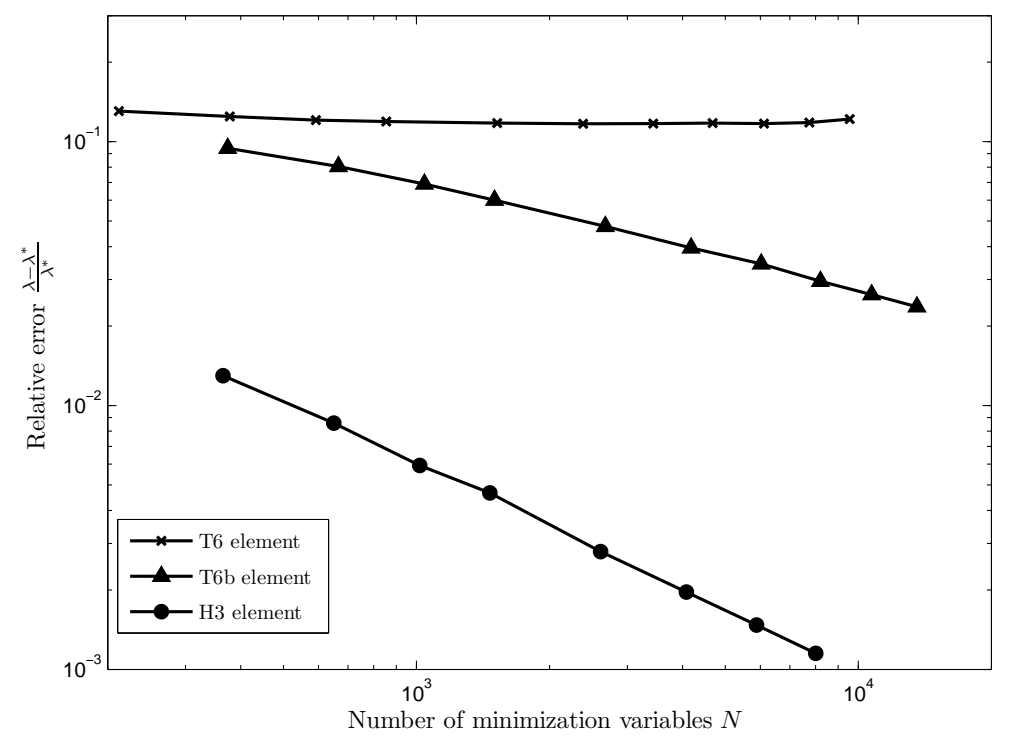

Figure 14: Relative error for the simply supported circular plate

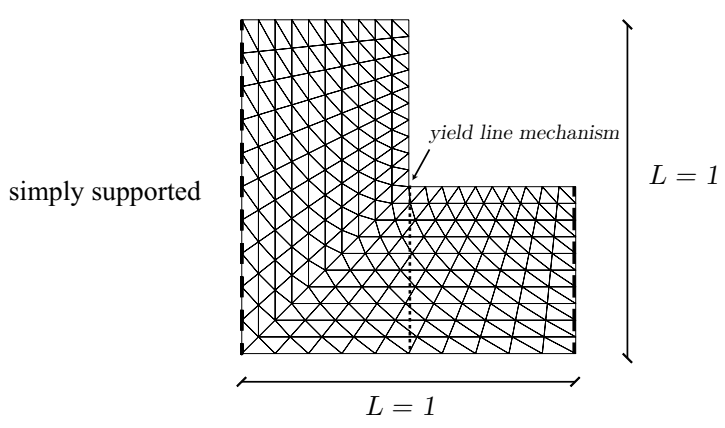

Figure 15: L-shaped plate problem and considered mesh with $M=10$ elements along edges 


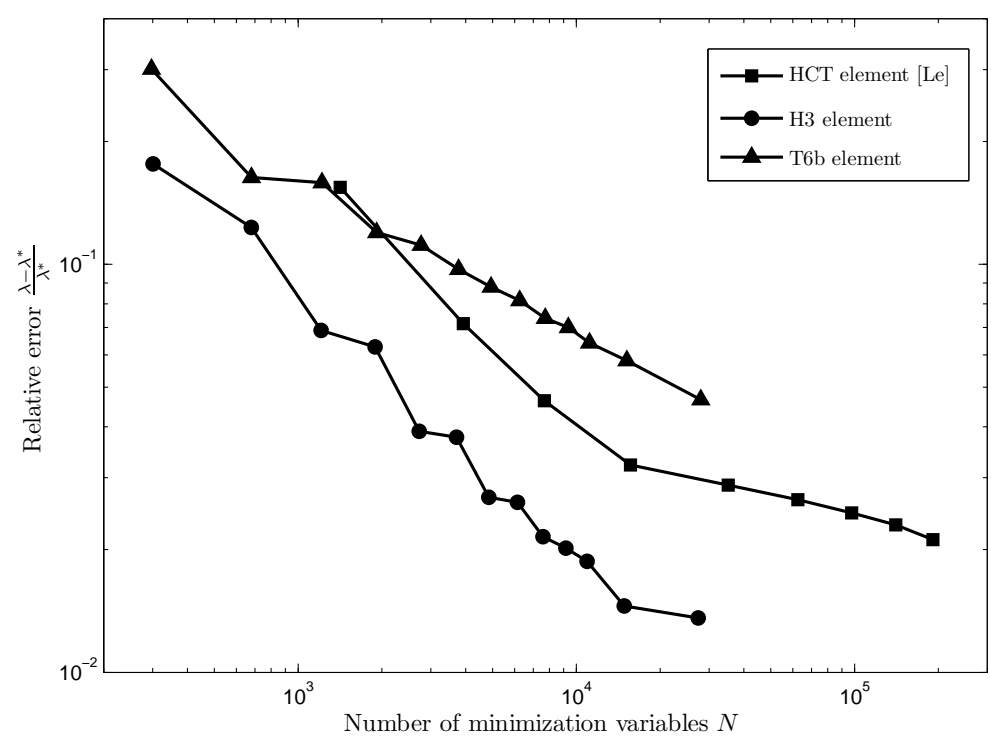

Figure 16: Relative error for the L-shaped plate

line theory by considering a yield line crossing the middle of the plate (Fig. 15). Calculations for this particular mechanism give an upper bound of $\lambda^{*}=\frac{32}{3 \sqrt{3}} \approx 6.16$. Computed bounds were compared to this reference value.

Convergence analysis in Figure 16 shows, in this case, that the performance of the HCT element is better than in the previous examples. We see, in particular, that T6b is as accurate as HCT for low values of $N$ but its convergence rate is smaller than HCT. H3 still performs well although being closer to HCT in terms of performance. Best obtained values are $\lambda=6.44$ for T6b ( $4.7 \%$ of error), $\lambda=6.29$ for $\operatorname{HCT}$ (2.1\% of error) and $\lambda=6.24$ for $\mathrm{H} 3$ (1.4\% of error).

To conclude with this example, it is interesting to note that the mesh used for this problem was not anticipating any known failure mechanism. Thus, even for a general mesh layout, T6b and H3 perform quite well in comparison to a fully conforming element. However, if the finite element mesh had been displayed so as to favour the previous yield line mechanism, performances of T6b and $\mathrm{H} 3$ would have been much better than fully conforming elements since rotation discontinuities are taken into account with such elements.

\section{Concluding remarks and future work}

An efficient numerical method and related computational tool have been set up for deriving accurate upper bounds to the limit loads of bending plates. It is based upon two decisive ingredients :

- The minimization procedure is treated as a second order cone programming (SOCP) problem for which a dedicated software package, already successfully employed for solving limit analysis or yield design problems, is available.

- The second, and probably most decisive, ingredient is the use of non-conforming finite elements for generating the velocity fields involved in the upper bound kinematic approach. Such elements incorporate not only curvature-type deformations as in f.e.m.-based elastic analysis, but also rotation discontinuities across the elements edges, which are perfectly admissible in the framework of the yield design/limit analysis approach. 
In particular, it has been shown on some relevant benchmark examples that the use of triangular "bubble" elements (T6b) and even more of cubic Hermite triangles (H3), combined with the SOCP minimization procedure, produces excellent results both in terms of numerical accuracy and computational time saving, when compared to previous numerical methods.

The same kind of improvement should be undertaken for plates where the strength properties are not only formulated in terms of bending moments but also of membrane or even shear forces. This is obviously the case of multilayered or composite plates (such as reinforced concrete slabs), the strength of which can be captured by means of interaction formulas. The corresponding upper bound kinematic approach must then be generalized by considering more complex velocity fields than those pertaining to bending plates, leading for instance to in-plane deformations to be combined with curvatures. Specific developments regarding the most appropriate finite elements to be employed in such a situation can be foreseen in the near future.

\section{Acknowledgements}

The authors would like to thank the Mosek software package team for their free release policy for academic research.

\section{References}

[1] Salençon J. Calcul à la rupture et analyse limite. Presses de l'Ecole Nationale des Ponts et Chaussées, 1983.

[2] Salençon J. Handbook of Continuum Mechanics: General Concepts, Thermoelasticity. Physics and Astronomy Online Library, Springer, 2001.

[3] Johansen K. Yield-line theory. Cement and Concrete Association London, 1962.

[4] Sloan S. Lower bound limit analysis using finite elements and linear programming. International Journal for Numerical and Analytical Methods in Geomechanics 1988; 12(1):61-77.

[5] Krabbenhoft K, Damkilde L. Lower bound limit analysis of slabs with nonlinear yield criteria. Computers $\&$ structures 2002; 80(27):2043-2057.

[6] Ciria H, Peraire J, Bonet J. Mesh adaptive computation of upper and lower bounds in limit analysis. International journal for numerical methods in engineering 2008; 75(8):899-944.

[7] Sloan S. Upper bound limit analysis using finite elements and linear programming. International Journal for Numerical and Analytical Methods in Geomechanics 1989; 13(3):263-282.

[8] Pastor J, Thai TH, Francescato P. New bounds for the height limit of a vertical slope. International Journal for Numerical and Analytical Methods in Geomechanics 2000; 24(2):165 - 182.

[9] Makrodimopoulos A, Martin C. Upper bound limit analysis using simplex strain elements and second-order cone programming. International journal for numerical and analytical methods in geomechanics 2007; 31(6):835-865.

[10] Le C, Gilbert M, Askes H. Limit analysis of plates using the efg method and second-order cone programming. International Journal for Numerical Methods in Engineering 2009; 78(13):15321552.

[11] Le C. Novel numerical procedures for limit analysis of structures: Mesh-free methods and mathematical programming. PhD Thesis, University of Sheffield 2010. 
[12] Zhou S, Liu Y, Chen S. Upper bound limit analysis of plates utilizing the c1 natural element method. Computational Mechanics 2012; 49:1-19.

[13] Mosek. The mosek optimization toolbox for matlab manual 2008. URL http://www.mosek.com/

[14] Christiansen E, Andersen K. Computation of collapse states with von mises type yield condition. International Journal for Numerical Methods in Engineering 1999; 46(8):1185-1202.

[15] Le C, Nguyen-Xuan H, Nguyen-Dang H. Upper and lower bound limit analysis of plates using fem and second-order cone programming. Computers \& Structures 2010; 88(1-2):65-73.

[16] Johnson D. Mechanism determination by automated yield-line analysis. Structural Engineer 1994; 72:323-323.

[17] Jennings A. On the identification of yield-line collapse mechanisms. Engineering structures 1996; 18(4):332-337.

[18] Thavalingam A, Jennings A, McKeown J, Sloan D. A computerised method for rigid-plastic yield-line analysis of slabs. Computers $\&$ structures 1998; 68(6):601-612.

[19] Bræstrup M. Yield-line theory and limit analysis of plates and slabs. Danmarks Tekniske Højskole, Afdelingen for Bærende Konstruktioner, 1971.

[20] Hodge Jr P, Belytschko T. Numerical methods for the limit analysis of plates. Journal of Applied Mechanics 1968; 35:796.

[21] Capsoni A, Corradi L. Limit analysis of plates- a finite element formulation. Structural Engineering and Mechanics 1999; 8(4):325-341.

[22] Zienkiewicz O, Taylor R, Taylor R. The finite element method for solid and structural mechanics, vol. 2. Butterworth-Heinemann, 2005.

[23] Ciarlet P. The finite element method for elliptic problems, vol. 4. North Holland, 1978.

[24] Munro J, Da Fonseca A. Yield line method by finite elements and linear programming. Structural Engineer 1978; 56(2):37-44.

[25] Balasubramanyam K, Kalyanaraman V. Yield-line analysis by linear programming. Journal of Structural Engineering 1988; 114(6):1431-1437.

[26] Makrodimopoulos A. Remarks on some properties of conic yield restrictions in limit analysis. International Journal for Numerical Methods in Biomedical Engineering 2010; 26(11):1449-1461.

[27] Clough R, Tocher J. Finite element stiffness matrices for analysis of plates in bending. Proceedings of conference on matrix methods in structural analysis, vol. 1, 1965.

[28] Fox E. Limit analysis for plates: the exact solution for a clamped square plate of isotropic homogeneous material obeying the square yield criterion and loaded by uniform pressure. Philosophical Transactions of the Royal Society of London. Series A, Mathematical and Physical Sciences 1974; 277(1265):121-155.

[29] Andersen KD, Christiansen E, Overton ML. Computing limit loads by minimizing a sum of norms. SIAM Journal on Scientific Computing 1998; 19(3):1046-1062.

[30] Save M, Massonnet C, de Saxce G. Plastic limit analysis of plates, shells, and disks, vol. 43. North Holland, 1997. 


\section{A Shape functions and derivatives}

\section{A.1 T6 element}

The following shape functions are used for the T6 element :

$$
\begin{array}{ll}
N_{1}(\xi, \eta)=\xi(2 \xi-1) & N_{4}(\xi, \eta)=4 \xi \eta \\
N_{2}(\xi, \eta)=\eta(2 \eta-1) & N_{5}(\xi, \eta)=4 \eta(1-\xi-\eta) \\
N_{3}(\xi, \eta)=(1-\xi-\eta)(1-2 \xi-2 \eta) & N_{6}(\xi, \eta)=4 \xi(1-\xi-\eta)
\end{array}
$$

Matrices for first and second derivatives are :

$$
\begin{gathered}
{\left[D_{1}(\xi, \eta)\right]=\left[\begin{array}{cccccc}
4 \xi-1 & 0 & -3+4 \xi+4 \eta & 4 \eta & -4 \eta & 4-8 \xi-4 \eta \\
0 & 4 \eta-1 & -3+4 \xi+4 \eta & 4 \xi & 4-4 \xi-8 \eta & -4 \xi
\end{array}\right]} \\
{\left[D_{2}(\xi, \eta)\right]=\left[\begin{array}{cccccc}
4 & 0 & 4 & 0 & 0 & -8 \\
0 & 4 & 4 & 0 & -8 & 0 \\
0 & 0 & 8 & 8 & -8 & -8
\end{array}\right]}
\end{gathered}
$$

\section{A.2 T6b element}

The following shape functions are used for the T6b element :

$$
\begin{array}{rlrl}
N_{1}(\xi, \eta) & =\xi(2 \xi-1) & N_{4}(\xi, \eta) & =4 \xi \eta \\
N_{2}(\xi, \eta) & =\eta(2 \eta-1) & N_{5}(\xi, \eta) & =4 \eta(1-\xi-\eta) \\
N_{3}(\xi, \eta) & =(1-\xi-\eta)(1-2 \xi-2 \eta) & N_{6}(\xi, \eta) & =4 \xi(1-\xi-\eta) \\
N_{7}(\xi, \eta)=27 \xi \eta(1-\xi-\eta) &
\end{array}
$$

Matrices for first and second derivatives are :

$$
\begin{gathered}
{\left[D_{1}(\xi, \eta)\right]=\left[\begin{array}{ccccccc}
4 \xi-1 & 0 & -3+4 \xi+4 \eta & 4 \eta & -4 \eta & 4-8 \xi-4 \eta & -27 \eta(2 \xi-1+\eta) \\
0 & 4 \eta-1 & -3+4 \xi+4 \eta & 4 \xi & 4-4 \xi-8 \eta & -4 \xi & -27 \xi(\xi-1+2 \eta)
\end{array}\right]} \\
{\left[D_{2}(\xi, \eta)\right]=\left[\begin{array}{cccccccc}
4 & 0 & 4 & 0 & 0 & -8 & -54 \eta \\
0 & 4 & 4 & 0 & -8 & 0 & -54 \xi \\
0 & 0 & 8 & 8 & -8 & -8 & 54-108 \xi-108 \eta
\end{array}\right]}
\end{gathered}
$$

\section{A.3 H3 element}

The following shape functions are used for the H3 element :

$$
\begin{aligned}
N_{1}(\xi, \eta) & =(1-\xi-\eta)\left(1+\xi+\eta-2 \xi^{2}-2 \eta^{2}-11 \xi \eta\right) \\
N_{2}(\xi, \eta) & =\xi(1-\xi-\eta)(1-\xi-2 \eta) \\
N_{3}(\xi, \eta) & =\eta(1-\xi-\eta)(1-2 \xi-\eta) \\
N_{4}(\xi, \eta) & =-2 \xi^{3}+7 \xi^{2} \eta+7 \xi \eta^{2}+3 \xi^{2}-7 \xi \eta \\
N_{5}(\xi, \eta) & =\xi^{3}-2 \xi^{2} \eta-2 \xi \eta^{2}-\xi^{2}+2 \xi \eta \\
N_{6}(\xi, \eta) & =\xi \eta(\eta+2 \xi-1) \\
N_{7}(\xi, \eta) & =7 \xi^{2} \eta+7 \xi \eta^{2}-2 \eta^{3}+3 \eta^{2}-7 \xi \eta \\
N_{8}(\xi, \eta) & =\xi \eta(\xi+2 \eta-1) \\
N_{9}(\xi, \eta) & =\eta^{3}-2 \xi^{2} \eta-2 \xi \eta^{2}-\eta^{2}+2 \xi \eta \\
N_{10}(\xi, \eta) & =27 \xi \eta(1-\xi-\eta)
\end{aligned}
$$




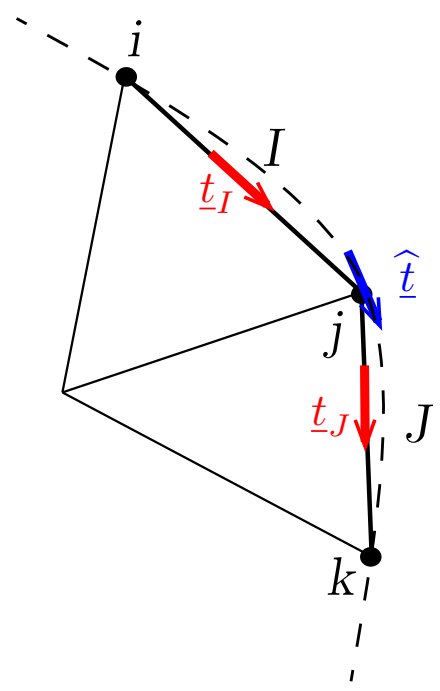

Figure 17: Tangent average strategy for boundary conditions on curved boundaries

Matrices for first and second derivatives are :

$$
T\left[D_{1}(\xi, \eta)\right]=\left[\begin{array}{cc}
-6 \xi^{2}+14 \xi \eta+7 \eta^{2}+6 \xi-7 \eta & 7 \xi(-1+\xi+2 \eta) \\
3 \xi^{2}-4 \xi \eta-2 \eta^{2}-2 \xi+2 \eta & -2 \xi(-1+\xi+2 \eta) \\
\eta(\eta-1+4 \xi) & \xi(-1+2 \xi+2 \eta) \\
7 \eta(-1+2 \xi+\eta) & 7 \xi^{2}+14 \xi \eta-6 \eta^{2}+6 \eta-7 \xi \\
\eta(-1+2 \xi+2 \eta) & \xi(\xi+4 \eta-1) \\
-2 \eta(-1+2 \xi+\eta) & 3 \eta^{2}-2 \xi^{2}-4 \xi \eta-2 \eta+2 \xi \\
-6 \xi-13 \eta+6 \xi^{2}+13 \eta^{2}+26 \xi \eta & -13 \xi-6 \eta+13 \xi^{2}+6 \eta^{2}+26 \xi \eta \\
1-4 \xi-3 \eta+3 \xi^{2}+6 \xi \eta+2 \eta^{2} & -3 \xi+3 \xi^{2}+4 \xi \eta \\
\eta(3 \eta-3+4 \xi) & 1-3 \xi-4 \eta+2 \xi^{2}+6 \xi \eta+3 \eta^{2} \\
27 \eta(1-\xi-\eta)-27 \xi \eta & 27 \xi(1-\xi-\eta)-27 \xi \eta \\
T\left[D_{2}(\xi, \eta)\right]=\left[\begin{array}{ccc}
-12 \xi+14 \eta+6 & 14 \xi & 28 \xi+28 \eta-14 \\
6 \xi-4 \eta-2 & -4 \xi & -8 \xi-8 \eta+4 \\
4 \eta & 2 \xi & -2+8 \xi+4 \eta \\
14 \eta & 14 \xi-12 \eta+6 & 28 \xi+28 \eta-14 \\
2 \eta & 4 \xi & -2+4 \xi+8 \eta \\
-4 \eta & 6 \eta-4 \xi-2 & -8 \xi-8 \eta+4 \\
-6+12 \xi+26 \eta & -6+12 \eta+26 \xi & -26+52 \eta+52 \xi \\
-4+6 \xi+6 \eta & 4 \xi & -6+12 \xi+8 \eta \\
4 \eta & -4+6 \xi+6 \eta & -6+8 \xi+12 \eta \\
-54 \eta & -54 \xi & 54-108 \xi-108 \eta
\end{array}\right]
\end{array}\right.
$$

\section{B Special treatment of curved boundaries for $\mathrm{H} 3$ element}

Since the H3 element involves partial derivatives as degree of freedom, special attention must be taken when dealing with curved boundaries.

Let consider two edges $I$ (nodes $i-j$ ) and $J$ (nodes $j-k$ ) on a boundary (figure 17). If the normal rotation $\partial_{n} u=0$ on the boundary, its contribution is added to the maximum resisting work. If $\partial_{n} u$ 
is free on the boundary, its contribution is zero.

However, the condition $u=0$ on the boundary implies specific conditions on corresponding nodal values. Since $u$ is cubic on an edge, $u_{i}=u_{j}=0$ does not suffice to impose $u=0$ on the whole edge $I$. A first solution is to impose two more conditions on the tangential derivatives on each element namely $\partial_{t_{I}} u_{i}=0$ and $\partial_{t_{I}} u_{j}=0$ which are the tangent rotations at each node on element $I$.

However, when applying the same procedure to element $J$ (for which the tangent vector is different from the one on $I$ ), two different conditions are imposed at node $j$ :

$$
\partial_{t_{I}} u_{j}=0 \quad \text { and } \quad \partial_{t_{J}} u_{j}=0
$$

Since $\underline{t}_{I} \neq \underline{t}_{J}$, these conditions restrain the tangent rotation as well as the normal rotation although it is not desired. This boundary condition is too restrictive and leads to poor upper bounds estimates. As a consequence, the following strategy was adopted (Fig. 17) : instead of (20), the following average condition was imposed at node $j$ :

$$
\partial_{\hat{t}} u_{j}=0 \quad \text { where } \quad \underline{\underline{t}}=\frac{1}{2}\left(\underline{t}_{I}+\underline{t}_{J}\right)
$$

This strategy was found to give very good results whereas its implementation remains very simple. Finally, let remark that this averaging strategy was not performed on corner points on the true geometry (e.g. the four corners of the square plate), since for these points both rotations have to be restrained. 